\title{
Los predictores de satisfacción de pacientes en un centro hospitalario universitario
}

\author{
Luis Castillo, Alberto Dougnac, Irene Vicente ${ }^{a}$, Víctor Muñoz ${ }^{b}$, \\ Víctor Rojas. \\ Predictors of the level of patient \\ satisfaction in a university hospital
}

\begin{abstract}
Background: Client satisfaction is an important indicator of the quality of services, and health care is not an exemption. Aim: To explore and establish the predictors that exert the greater influence on the level of satisfaction with the service offered in a University Hospital. Material and method: The source of the study is a data base, obtained from a survery made to 355 patients of the Hospital Clínico de la Pontificia Universidad Católica de Chile prior to discharge. A multiple regression analysis was used to determine the relative importance of the different factors in the quality of service. The regression variables were reduced to a number of factors, obtained of Factorial Analysis and a Varimax rotation, allowing them to clarify their relations. Results: After performing the main components analysis, seven factors or dimensions that explained a $69 \%$ of the total variance were obtained. The predictive model explains up to $40 \%$ of the criterion variable (Global Satisfaction during the hospitalization). The factor with the greatest predictive power was the relationship between the patients and nursing staff. Other factors that emerged as important were communication, medical attention and room conditions. Conclusions: Perceptive type components had the greater relative weight to determine the level of patient satisfaction in this survey (Rev Méd Chile 2007; 135: 696-701).
\end{abstract}

(Key words: Delivery of health care; Patients care management; Patient satisfaction)
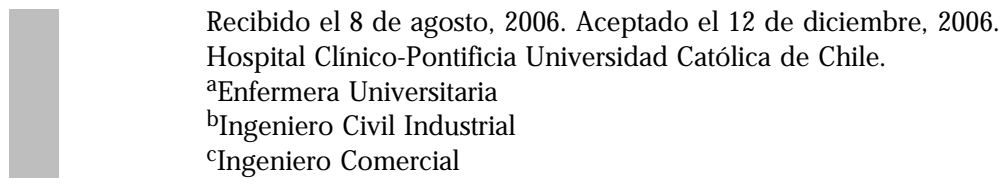

$\mathrm{U}$ n mayor nivel de satisfacción del cliente reforzará sus percepciones de calidad ${ }^{1}$.

La calidad es uno de los elementos estratégicos en que se fundamenta la transformación y mejora de los sistemas de salud modernos. El estudio de

Correspondencia a: Dr. Luis Castillo. Marcoleta 367, Santiago de Chile. Fono 3546426. E mail: castillo@med.puc.cl

la calidad en salud supone significados distintos para los pacientes, profesionales y gestores.

La satisfacción del cliente es un indicador importante de la calidad de servicios, incluso, algunos autores consideran calidad y satisfacción como sinónimos. Liljander sugiere que los modelos de satisfacción pueden ser denominados de calidad de servicio percibida, ya que lo que se estudia es un servicio y no un bien de consumo ${ }^{2}$. 
La satisfacción con los servicios de salud es un concepto complejo que está relacionado con una gran variedad de factores, como son el estilo de vida, las experiencias previas, las expectativas de futuro y los valores del individuo y de la socie$\operatorname{dad}^{3}$.

Desde la perspectiva del paciente, la tarea del médico se puede dividir en: la técnica (ciencia) y la interpersonal. A estas dos tareas habría que añadir las condiciones bajo las cuales se ofrece la atención, tales como el confort, aspectos relativos a la información o comunicación, la rapidez o la amabilidad $^{4}$.

La medida de la satisfacción será, entonces, la medida de las diferentes dimensiones que la conforman ${ }^{5} \mathrm{y}$ ha demostrado ser un instrumento útil para evaluar las intervenciones de los servicios de salud, porque proporciona información sobre la calidad percibida 6 y facilita información a los profesionales, gestores y administradores sobre aquellos aspectos de la organización sanitaria, percibidos como insatisfactorios y que son susceptibles de mejoría.

La idea de que el éxito de una organización depende de la capacidad de satisfacer las necesidades de sus clientes, se ha generalizado hasta tal punto que se ha llegado a modificar la definición de calidad. Por este motivo es imprescindible tener perfectamente definido el concepto de satisfacción de clientes, desarrollando sistemas de evaluación y gestionando la insatisfacción, con el objetivo de lograr lealtad o fidelidad de los clientes.

Actualmente, una institución de salud que esté interesada en garantizar la efectividad de la atención sanitaria y eficiencia en la gestión, debe conocer las percepciones de los pacientes respecto a los procesos asistenciales.

Este hecho exige determinar y supervisar parámetros de calidad relacionados con infraestructura, equipamiento, procesos y personal vinculados a la atención de salud prestada, con el fin de lograr la mayor satisfacción de los pacientes.

Considerando que las variables relacionadas con la satisfacción son multidimensionales, se utilizará el análisis factorial a objeto de simplificar las numerosas y complejas relaciones que se puedan encontrar en el conjunto de variables cuantitativas observadas.

El objetivo de este estudio es explorar y establecer los predictores significativos del servi- cio prestado, que ejercen la mayor influencia en el nivel de satisfacción de los clientes (pacientes), encontrando las dimensiones o factores que ponen en relación a las aparentemente no relacionadas variables. Esta información contribuirá a establecer una base para las mejoras en nuestro centro asistencial, con la consecuente mayor satisfacción de nuestros clientes.

\section{Metodología DE ANÁLISIS}

En este estudio, se encuestaron pacientes hospitalizados en el Hospital Clínico de la Pontificia Universidad Católica de Chile y que cumplieron los siguientes criterios de elegibilidad: aceptar participar en el estudio, previa información de las características del estudio, ser mayores de 15 años de edad y previo al alta.

Para alcanzar el objetivo propuesto se realizaron ordenadamente las siguientes fases:

Se aplicó una encuesta entre agosto y septiembre de 2004, el cuestionario incluyó un área de clasificación demográfica (edad, sexo, previsión y tipo de ingreso, por urgencia o programado) y 30 preguntas que indagan sobre diferentes perspectivas de determinados atributos de los servicios asistenciales y una de ellas dirigida a obtener una opinión sobre el nivel general de satisfacción, la que es utilizada como variable respuesta (Nivel de satisfacción general con la atención), utilizando un formato de entrevista semiestructurada. En cada uno de los aspectos se utilizó una escala de Likert de 0 a 5 donde 0 significó nada satisfecho»y 5 totalmente satisfecho», en cada uno de sus aspectos.

El muestreo fue aleatorio, estratificado con distribución proporcional a cada estrato (número de camas y nivel de ocupación por servicio). El número de observaciones para el estudio, se calculó a partir de la fórmula para variables cualitativas.

$$
\mathrm{n}=\mathrm{Z}^{2} \frac{[\mathrm{p}(1-\mathrm{p})]}{\mathrm{I}^{2}}
$$

El tamaño n, se calculó tomando en consideración un error permitido de 5\% (I) para un nivel de confianza de $95 \%(Z=1,96)$ y una proporción de personas con un nivel de percepción satisfactorio de, al menos, $65 \%$ (p). 
El total encuestado (n) fueron 355 pacientes/ clientes del hospital, de un universo de 1.800.

Con los datos obtenidos, se realizó un análisis exploratorio que permite obtener un indicador de fiabilidad alpha de Crombach, cuyo valor de 0,8547 es suficiente y permite el análisis exploratorio.

Un análisis factorial con el objeto de simplificar las relaciones encontradas en el conjunto de variables cuantitativas observadas y posteriormente, un análisis de regresión múltiple para determinar el impacto de esos factores en la satisfacción con el servicio. El análisis de componentes principales (análisis factorial) realizado, explica 69\% de la varianza en 7 variables subyacentes o factores». Las variables reducidas son trabajadas como puntuaciones factoriales.

Posteriormente, se realizó un análisis de regresión múltiple con método stepwise para determinar el impacto en la satisfacción global con el servicio. Para éste, se fijó una probabilidad $F$ para entrar menor 0 igual a 0,05, y una probabilidad F para salir de 0,1.

El análisis estadístico fue realizado con el programa SPSS 7.5.

Ventajas y limitaciones. La utilización del análisis de factores permitió reducir el número de variables a un conjunto más manejable, reteniendo la mayor información posible. Los resultados de un análisis factorial, simplemente ponen de manifiesto un conjunto de factores comunes cuyo significado se deduce de las cargas factoriales, sin embargo, toda interpretación de éstos debe ser validada por algún criterio externo.

\section{PRESENTACIÓN DE RESULTADOS}

Características generales de los entrevistados. Sesenta y tres por ciento de los encuestados eran de sexo femenino, y el 37\% restante masculino. El porcentaje de los entrevistados por edades fue: $25 \%$ entre 16 y 35 años, $49 \%$ entre 36 y 64 años y $25 \%$ mayor a 64 años.

Los encuestados tenían diferentes tipos de previsión: $40 \%$ afiliado a Fonasa, 52\% estaba afiliado a alguna Isapre y $7 \%$ restante no tenía previsión.

Obtención de elementos del servicio que incrementan la fiabilidad. Aplicado el análisis de fiabilidad, se eliminaron nueve ítems, de manera que los finalmente introducidos fueron 21. La escala de satisfacción del paciente con distintos elementos del servicio después de eliminar 9 de ellos (con el objetivo de incrementar la fiabilidad), arrojó un alpha de 0,8547. El valor del coeficiente fue suficiente para el análisis exploratorio.

Resultados del factorial exploratorio para la satisfacción del cliente. El estadístico de Kaiser-MeyerOlkin (KMO) o medida de adecuación muestral obtenido fue 0,785 con un Chi-cuadrado aproximado de 1810,5 y significativo 0,000.

Al comparar las magnitudes de los coeficientes de correlación observados con las magnitudes de los coeficientes de correlación parciales puso de manifiesto la idoneidad de la aplicación, ya que la medida de adecuación muestral de Keiser-MeyerOlkin $(0,785)$ permite esperar un buen ajuste factorial y el test de esfericidad de Bartlett (significativo al $0,000 \%$ ) presentan valores que confirman la conveniencia del análisis factorial.

Realizado el análisis, se obtuvieron siete factores o dimensiones que explican prácticamente $70 \%$ de la varianza total (Tabla 1). La composición de los factores se encuentra en la Tabla 2.

Los significados de los factores arrojados se asociaron a:

Factor 1: «Alimentación durante la hospitalización». Este factor fue el que explicó un mayor porcentaje de varianza (12,3\%). Este factor define como ítems de mayor peso, los referidos a la presentación, variedad y temperatura de los alimentos ofrecidos.

Factor 2: «Condiciones de limpieza y comodidad en la habitación». Este factor explicó $11,5 \%$ de la varianza. Los ítems más destacados son los enunciados sobre el buen nivel de limpieza en la habitación y la comodidad de la cama.

Factor 3: «Relación del personal de enfermería con el paciente y las condiciones para el descanso». Explicó $11 \%$ de la varianza.

Factor 4: «Orientación a la necesidad de información y atención recibida del médico». Explicó un porcentaje de la varianza de $10 \%$.

Factor 5: $O$ rientación al paciente del personal de admisión», explicó 9\% de la varianza.

Factor 6: «Satisfacción ante requerimientos a enfermería» explicó 8,8\% de la varianza.

Factor 7: «atisfacción con la asistencia espiritual», explicó $6 \%$ de la varianza. 
Tabla 1. Factores o D imensiones de la satisfacción con los elementos del servicio y el \% de la Varianza Explicada

\begin{tabular}{|lccc|}
\hline $\begin{array}{l}\text { Componentes } \\
\text { principales }\end{array}$ & $\begin{array}{c}\text { Varianza } \\
\text { total }\end{array}$ & \% Varianza & $\begin{array}{c}\text { Varianza } \\
\text { acumulada \% }\end{array}$ \\
\hline Factor 1 & 2,581 & 12,292 & 12,292 \\
Factor 2 & 2,416 & 11,504 & 23,796 \\
Factor 3 & 2,334 & 11,113 & 34,909 \\
Factor 4 & 2,151 & 10,241 & 45,151 \\
Factor 5 & 1,896 & 9,029 & 54,18 \\
Factor 6 & 1,858 & 8,846 & 63,027 \\
Factor 7 & 1,296 & 6,172 & 69,198 \\
\hline
\end{tabular}

Fuente: Elaborado en SPSS v 7.5 (Matriz de componentes rotados) (Normalización Varimax).

Tabla 2. Variables que se incluyen en el factor

\begin{tabular}{|lrrrrrrr|}
\hline Variables & \multicolumn{7}{c|}{ Composición de los Factores } \\
& Factor & Factor & Factor & Factor & Factor & Factor & Factor \\
& 1 & 2 & 3 & 4 & 5 & 6 & 7 \\
\hline Presentación de la alimentación & 0,836 & 0,186 & 0,106 & 0,136 & 0,142 & $7,40 \mathrm{E}-02$ & $5,64 \mathrm{E}-02$ \\
Variedad en la alimentación & 0,820 & 0,318 & $9,48 \mathrm{E}-02$ & $-4,24 \mathrm{E}-02$ & $8,82 \mathrm{E}-02$ & $9,83 \mathrm{E}-02$ & $-3,79 \mathrm{E}-02$ \\
Temperatura de la alimentación & 0,760 & 0,142 & 0,113 & 0,227 & $8,70 \mathrm{E}-02$ & 0,144 & $2,34 \mathrm{E}-02$ \\
Aseo en pieza & 0,125 & 0,845 & 0,105 & 0,16 & 0,142 & $5,53 \mathrm{E}-02$ & $8,32 \mathrm{E}-04$ \\
Aseo baño & 0,195 & 0,837 & $9,94 \mathrm{E}-02$ & 0,215 & 0,102 & $-6,51 \mathrm{E}-03$ & $-1,43 \mathrm{E}-02$ \\
Comodidad de la cama & 0,233 & 0,586 & $9,83 \mathrm{E}-02$ & $1,71 \mathrm{E}-02$ & $4,60 \mathrm{E}-02$ & 0,209 & $5,25 \mathrm{E}-02$ \\
Nivel de ruidos por la actividad & & & & & & & \\
asistencial & $-0,101$ & $1,99 \mathrm{E}-02$ & 0,693 & $2,90 \mathrm{E}-02$ & $6,55 \mathrm{E}-02$ & 0,178 & $4,61 \mathrm{E}-02$ \\
Comunicación con las enfermeras & 0,308 & $8,68 \mathrm{E}-02$ & 0,672 & 0,368 & $-1,91 \mathrm{E}-02$ & 0,155 & $1,22 \mathrm{E}-02$ \\
Identificación del personal & $8,11 \mathrm{E}-02$ & 0,164 & 0,585 & $-8,24 \mathrm{E}-02$ & 0,172 & 0,255 & $-0,182$ \\
Trato del personal de enfermería & 0,311 & $3,34 \mathrm{E}-02$ & 0,555 & 0,499 & $4,31 \mathrm{E}-02$ & 0,106 & $3,28 \mathrm{E}-02$ \\
Descanso diurno & 0,28 & 0,431 & 0,544 & 0,119 & 0,152 & $-0,129$ & $3,86 \mathrm{E}-02$ \\
Descanso nocturno & 0,287 & 0,361 & 0,476 & 0,379 & 0,162 & $-8,21 \mathrm{E}-02$ & $1,96 \mathrm{E}-02$ \\
Información médica & $7,23 \mathrm{E}-03$ & 0,207 & 0,106 & 0,848 & 0,101 & $3,33 \mathrm{E}-02$ & $-5,01 \mathrm{E}-02$ \\
Atención médica & 0,178 & 0,137 & $8,31 \mathrm{E}-02$ & 0,793 & 0,18 & 0,112 & $-2,68 \mathrm{E}-02$ \\
Actitud y disposición en admisión & 0,139 & 0,137 & 0,134 & 0,135 & 0,906 & $1,51 \mathrm{E}-02$ & $4,50 \mathrm{E}-02$ \\
Disposición a respuestas en admisión & 0,126 & 0,157 & 0,113 & 0,157 & 0,905 & $9,82 \mathrm{E}-02$ & $2,89 \mathrm{E}-02$ \\
Respuesta a los llamados & $5,46 \mathrm{E}-02$ & 0,166 & 0,132 & $9,38 \mathrm{E}-02$ & $-5,62 \mathrm{E}-03$ & 0,798 & $-5,38 \mathrm{E}-02$ \\
Manejo del dolor & $7,92 \mathrm{E}-02$ & $6,63 \mathrm{E}-02$ & 0,107 & $-4,30 \mathrm{E}-02$ & $-4,96 \mathrm{E}-03$ & 0,776 & 0,171 \\
Información de procedimientos de & & & & & & & \\
enfermería & 0,196 & $-0,102$ & 0,176 & 0,208 & 0,206 & 0,571 & $-0,192$ \\
Atención capellán & 0,17 & $-8,15 \mathrm{E}-03$ & 0,164 & $-0,106$ & 0,122 & $-0,123$ & 0,787 \\
Servicio religiosas & $-0,11$ & $4,24 \mathrm{E}-02$ & $-0,174$ & $4,31 \mathrm{E}-02$ & $-4,61 \mathrm{E}-02$ & 0,124 & 0,745 \\
\hline
\end{tabular}

Análisis de regresión de los factores de satisfacción del paciente. Una vez determinada la existencia de los componentes o factores principales, y aceptada la fiabilidad de las escalas subyacen- tes, se procedió a determinar sus potenciales efectos sobre los niveles de satisfacción, a través de un análisis de regresión múltiple con método stepwise. 
Variable respuesta: La satisfacción final con la atención de salud del paciente. Variable explicativa: Los componentes o factores previamente determinados, a través del análisis factorial aplicado anteriormente.

Los resultados obtenidos (Tabla 3), indican los factores analizados que ejercieron un efecto positivo y estadísticamente significativo $(\mathrm{p}<0,05)$ sobre el nivel de satisfacción.

Los términos residuales obtenidos a partir de la regresión fueron normalizados, rechazando Ho con un nivel de significación de $\alpha=5 \%$, los individuos que cumplieron con esta condición se identificaron como outliers, detectándose 6 y se eliminaron.

La predicción de la satisfacción global durante la hospitalización, fue explicada en gran medida por los 6 primeros factores analizados, fueron éstos los que ejercen un efecto positivo con una significación $(p<0,05)$.

\section{DiSCUSIÓN}

El análisis aplicado a los distintos aspectos de la atención y el servicio prestado por el hospital, ha extraído los aspectos más relevantes para explicar el grado de satisfacción de los pacientes. De todos los componentes seleccionados, los de carácter perceptivo aportan mayor peso relativo en la determinación de la satisfacción general con la atención, siendo la relación del personal de enfermenía con el paciente y las condiciones para el descanso los más importantes, seguido por la orientación a las necesidades de información y atención recibida del médico, los aspectos que el hospital debe potenciar para aumentar la satisfacción de los pacientes ${ }^{6-10}$.

El estudio se realizó en un establecimiento universitario privado, sin embargo, sus resultados indican cierta universalidad, ya que los factores identificados son coincidentes con otros estudios de centros hospitalarios realizados internacionalmente ${ }^{9}$.

Tabla 3. Análisis de regresión de los factores de orientación hacia la satiffacción del paciente

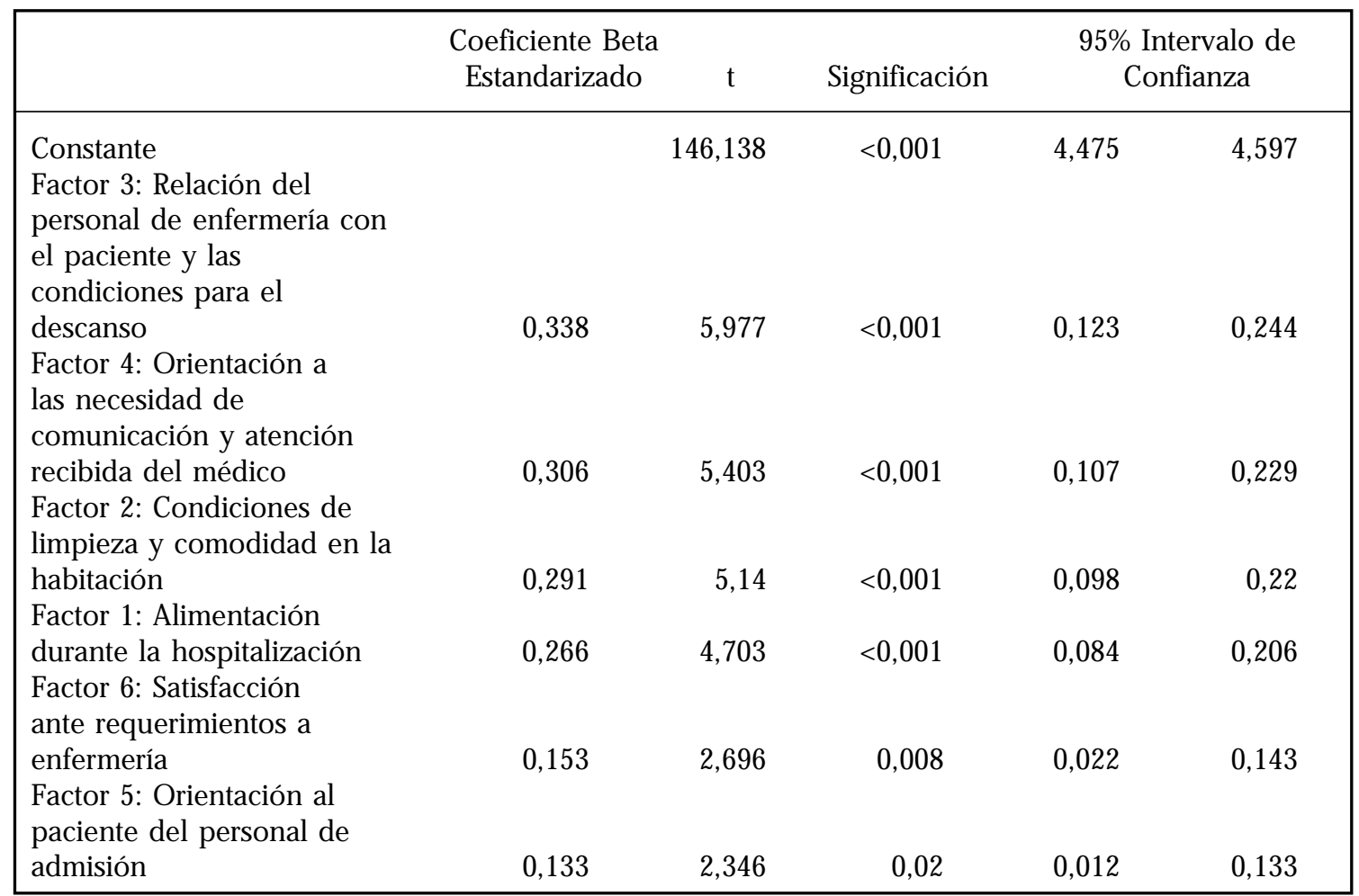

$\mathrm{R}^{2}=0,404 ; \mathrm{R}^{2}$ ajustado $=0,382$. 
El análisis de los factores nos lleva a las siguientes conclusiones:

A nivel de atención, la característica que proporciona una mayor influencia por sobre las condiciones de confort y alimentación en la satis-

\section{REFERENCIAS}

1. BerNé C, Múgica JM, Yagüe MJ. La gestión estratégica y los conceptos de calidad percibida, satisfacción del cliente y lealtad. Economía Industrial 1996; 307: 63-74.

2. LLJANDER V. Comparison standards in perceived service quality. Ekonomioch Samhälle, № 63. Publications of the Swedish School of Economics and Business Administration, Helsinki, Finland 1995.

3. CARR-HiLl RA. The measurement of patient satisfaction. J Public Health Med 1992; 14: 236-49.

4. Bolívar I, Grupo de estudio de la Utiluzación de SERvicios sanitarios de Mataró. Determinantes poblacionales de la satisfacción con el médico de atención primaria. Gac Sanit 1999; 13: 371-83. facción del paciente durante su hospitalización, es la relación interpersonal con el personal de enfermería, las condiciones que le permitan el descanso y los aspectos relacionados con la atención y comunicación del paciente con los médicos.

5. Donabedian A. Evaluating the quality of medical care. MMFQ 1966; 44: 166-206.

6. VuonI H. Patient satisfaction-An attribute or indicator of the quality of care? Quality Review Bulletin 1987; 13: 106-8.

7. NARESH K MalHotra. Investigación de Mercados. Prentice-Hall Hispanoamérica S.A. 1997; 644-69.

8. AAKER DA, DAY GS. Investigación de Mercados 1989 3a edición.

9. Monteagudo O, Navarro C, Alonso P, Casas R, Rodríguez L, Gracia J. Aplicación hospitalaria del SERVQHOS: factores asociados a la satisfacción e insatisfacción. Rev Calidad Asistencial 2003; 18: 263-71.

10. VARo J. Gestión Estratégica de la calidad en los Servicios Sanitarios 1994, Editorial Díaz de Santos, Madrid, España. 